\title{
Editorial
}

\section{The role of diet and nutrition on mental health and wellbeing}

\begin{abstract}
Mental, neurological and substance-use disorders presently represent the greatest global burden of disease. Likewise, depression and other psychopathologies are elevated risk comorbidities of other health hazards, such as obesity. Nutrition has been implicated in behaviour, mood and in the pathology and treatment of mental illness. In this brief editorial, we aim to set the scale of the problem in context and overview advances and recent evidence linking nutrition to psychological outcomes. The purpose of the 2016 Nutrition Society Winter Meeting, 'Diet, nutrition and mental health and wellbeing' was to review where the evidence is strong, where there are unmet needs for research and to draw together the communities working in this area to share their findings. The papers presented demonstrated clear advancements that are being made in this field. The meeting illustrated compelling support for nutrition as a modifiable risk factor. The present research in the field and evidence presented at the 2016 Nutrition Society Winter Meeting lead us to postulate that even interventions with relatively modest effect sizes may plausibly and significantly curtail the disease burden of mental and neurological disease by food- and nutrient-based approaches.
\end{abstract}

Diet: Nutrition: Mental health: Cognition: Wellbeing

\section{Mental, neurological and substance-use disorders are the leading global burden of disease}

It has recently been estimated that mental, neurological and substance-use disorders (i.e. schizophrenia, depression, epilepsy, dementia, alcohol dependence) account for $13 \%$ of the global burden of disease placing mental illness as the greatest burden, exceeding both CVD and cancer ${ }^{(1)}$. While sobering, these figures are perhaps unsurprising since there is now one new case of dementia diagnosed every $4 \mathrm{~s}$, or 7.7 million cases per year ${ }^{(2)}$. More than 300 million people are now living with depression, an increase of more than $18 \%$ between 2005 and 2015. By 2020, it is estimated that between 15 and 30 million people will attempt suicide and approximately 1.5 million per year will die by suicide each year ${ }^{(3)}$. Alcohol and illicit drug use account for over $5 \cdot 1 \%$ of global burden of disease and worldwide, 3.3 million deaths every year result from harmful use of alcohol ${ }^{(4)}$.

From social and economic perspectives, our health systems are not prepared for this extraordinary global burden. Lobbying for legislative and community-level interventions has begun and a World Health Assembly resolution passed in May 2013 has called for a comprehensive, coordinated response to mental disorders at country level. While treatment intervention is urgently needed, health improvements and cost savings are achievable by providing targeted, evidence-based, disease prevention programmes that reduce modifiable risk factors.

\section{Diet and nutrition as preventative measures}

Nutritional psychiatry is a new, rapidly emerging field of nutrition and mental health and is one such avenue of prevention. Over the past decade, there has been a steady increase in epidemiological studies investigating the relationships between dietary patterns and mental states. Both cross-sectional and longitudinal studies have shown that the more one eats a Western or highly processed diet, the more one is at risk for developing psychiatric symptoms, such as depression and anxiety. Conversely, the more one eats a Mediterranean-style diet, the more one is protected from developing a mental disorder ${ }^{(5)}$. In terms of direction of causality, in several studies, the dietary pattern has been shown to precede the onset of psychiatric symptoms. One randomised controlled trial published this year in $B M C$ Medicine demonstrated quite striking effects of a 3-month dietary intervention on moderate-to-severe depression, with a significantly greater improvement in the dietary intervention group and remission achieved in $32 \%$ of this group $^{(6)}$.

The consequences of a poor diet also impact on a further global health hazard: obesity. Alzheimer's disease and depression are comorbidities of obesity, leading to the theory that vascular impairments may have a role in the development of dementia and psychiatric pathologies. As a result of these observations, Alzheimer's disease and other dementias are now being thought of as potentially preventable diseases. In a 27 -year longitudinal study, obesity in mid-life was shown to double the risk of developing dementia at a later age ${ }^{(7)}$; moreover while cognitive deficits have been associated with glycaemic regulation in diabetes, only in the recent years has it been observed that cognitive deficits exist in young healthy, normal weight individuals with poor glucoregulation, again exemplifying the need for early, rather than later life, preventative nutrition measures. 
In addition to whole dietary and intervention strategies, significant advances have also been made in the study of nutraceuticals. Previous research in this area has been badged as 'complementary medicine' and scientific rigor of research investigating these interventions has not always matched that of pharmaceutical research. However, good converging evidence from high-quality randomised controlled trials is now demonstrating effectiveness of a large number of nutraceuticals as moodmodifying and cognitive-enhancing agents in both clinical and healthy populations (a comprehensive review is beyond the scope of the editorial). Recent mechanistic work has indicated plausible, modifiable, cognitionrelated targets for nutraceuticals, including processes involved with systemic and central vascular function, inflammation, metabolism, central activation, improved neural efficiency and angiogenesis. The quantity of good, well-controlled clinical trials examining nutraceutical interventions has meant that nutraceuticals are increasingly being used and prescribed in psychiatric practice.

A further new avenue in nutritional psychiatry has been the eruption of research targeting the modulation of the gut microbiota (through probiotic and prebiotic foods and supplements) as a novel therapy for the treatment of various neuropsychiatric conditions. The bidirectional communication between the gut microbiota and the brain has been shown to influence neurotransmission and the behaviour that is often associated with neuropsychiatric conditions, and likewise the microbiome has been associated with multiple systemic pathologies and obesity $^{(8)}$. However, this research is still in its infancy with an urgent need for prospective studies.

The mechanisms of nutritional impact on the brain are likely to be many, complex and compound. However, recent research has focused on adult hippocampal neurogenesis. The hippocampus is a brain region associated with learning, memory and mood and is one of two structures in the adult brain where neurogenesis persists. The degree of neurogenesis in the hippocampus has been linked directly to cognition and mood, thus modulation of hippocampal neurogenesis by diet has recently emerged as a possible mechanism by which nutrition may impact on brain plasticity, function and mental health ${ }^{(9)}$.

\section{Summary and final thoughts}

There have been some major advances in our understanding of the impact of nutrition on brain function and the aetiology and treatment of these disorders. Future breakthroughs are likely to combine nutritional genomics and neuroscience with psychological and psychiatric research. Due to the scale of the burden of mental, neurological and substance-use disorders and the universality of food as a modifiable risk factor, even small improvements in the nutritional environment may translate to large gains in mental health and wellbeing at a population level. Substantial research progress is being made in this field and the contemporary research outlining the evidence in relation to whole dietary interventions, nutraceuticals, alcohol use and putative mechanisms of action is presented in the present issue of Proceedings of the Nutritional Society in the form of a series of review papers authored by the speakers of the 2016 Nutrition Society Winter Meeting. However, future advancement is contingent upon appropriate investment and concerted action by mental health advocates, funding bodies, and policy makers, and the inclusion and recognition of the role of nutrition at the level of policymaking.

Lauren Owen ${ }^{1 *}$ and Bernard Corfe
1,3
Department of Psychology, University of Central
Lancashire, Darwin Building, Fylde Rd, Preston PRI
$2 H E, U K$
${ }^{2}$ Molecular Gastroenterology Research Group, Academic
Unit of Surgical Oncology, Department of Oncology \&
Metabolism, The Medical School, Beech Hill Road,
Sheffield, S10 2RX, UK
${ }^{3}$ Insigneo Institute for In Silico Medicine, The University
of Sheffield, UK
*Corresponding author: L. Owen,
email Dr.lauren.owen@gmail.com

\section{Acknowledgments}

None.

\section{Financial Support}

None.

\section{Conflicts of Interest}

None.

\section{Authorship}

None.

\section{References}

1. Vigo D, Thornicroft G \& Atun R. Estimating the true global burden of mental illness. Lancet Psychiatry 3, 171-178.

2. World Health Organization (2012) Dementia: a Public Health Priority. World Health Organization.

3. Bertolote JM \& Fleischmann A (2002) A global perspective in the epidemiology of suicide. Suicidology 7, 6-8.

4. World Health Organization (2014) Global Status Report on Alcohol and Health 2014. World Health Organization.

5. Jacka FN, Mykletun A \& Berk M (2012) Moving towards a population health approach to the primary prevention of common mental disorders. BMC Med 10, 149.

6. Jacka FN, O'Neil A, Opie R et al. (2017) A randomised controlled trial of dietary improvement for adults with major depression (the 'SMILES' trial). BMC Med 15, 23.

7. Whitmer RA, Gunderson EP, Barrett-Connor E et al. (2005) Obesity in middle age and future risk of dementia: a 27 year longitudinal population based study. BMJ 330, 1360.

8. Carding S, Verbeke K, Vipond DT et al. (2015) Dysbiosis of the gut microbiota in disease. Microbial Ecol Health Dis 26, 26191.

9. Stangl D \& Thuret S (2009) Impact of diet on adult hippocampal neurogenesis. Genes Nutr 4, 271-282. 